\title{
STATIC HEDGING OF BARRIER OPTIONS WITH A SMILE: AN INVERSE PROBLEM
}

\author{
Claude Bardos $^{1}$, Raphä̈l Douady ${ }^{2}$ and Andrei Fursikov ${ }^{3}$
}

\begin{abstract}
Let $L$ be a parabolic second order differential operator on the domain $\bar{\Pi}=[0, T] \times \mathbb{R}$. Given a function $\hat{u}: \mathbb{R} \rightarrow R$ and $\hat{x}>0$ such that the support of $\hat{u}$ is contained in $(-\infty,-\hat{x}]$, we let $\hat{y}: \bar{\Pi} \rightarrow \mathbb{R}$ be the solution to the equation:

$$
L \hat{y}=0,\left.\quad \hat{y}\right|_{\{0\} \times \mathbb{R}}=\hat{u} .
$$

Given positive bounds $0<x_{0}<x_{1}$, we seek a function $u$ with support in $\left[x_{0}, x_{1}\right]$ such that the corresponding solution $y$ satisfies:

$$
y(t, 0)=\hat{y}(t, 0) \quad \forall t \in[0, T] .
$$

We prove in this article that, under some regularity conditions on the coefficients of $L$, continuous solutions are unique and dense in the sense that $\left.\hat{y}\right|_{[0, T] \times\{0\}}$ can be $C^{0}$-approximated, but an exact solution does not exist in general. This result solves the problem of almost replicating a barrier option in the generalised Black-Scholes framework with a combination of European options, as stated by Carr et al. in [6].
\end{abstract}

Mathematics Subject Classification. 93C20, 65M32, 62P05, $91 \mathrm{~B} 28$.

Received July 19, 2001.

\section{INTRODUCTION}

\subsection{The inverse problem}

Let $L$ be a parabolic second order differential operator defined on the domain $\bar{\Pi}=[0, T] \times \mathbb{R}$ :

$$
L y=\partial_{t} y(t, x)-a_{2}(t, x) \partial_{x x} y(t, x)+a_{1}(t, x) \partial_{x} y(t, x)+a_{0}(t, x) y(t, x) .
$$

Given a function $u: \mathbb{R} \rightarrow R$ with mild growth: $u(x)=O\left(|x|^{m}\right)$, there is a unique solution $y: \bar{\Pi} \rightarrow \mathbb{R}$ to the equation:

$$
L y=0,\left.\quad y\right|_{\{0\} \times \mathbb{R}}=u .
$$

Keywords and phrases: Inverse problems, Carleman estimates, barrier option hedging, replication.

1 Université de Paris VII and C.M.L.A., URA 1611 du CNRS, École Normale Supérieure de Cachan, France.

2 C.M.L.A. 1611 URA du CNRS, École Normale Supérieure de Cachan, France; e-mail: Raphael@clearingrisk.com

3 Moscow State University. Partially supported by CNRS while visiting the Emile Borel Center, and by R.F.B.I. under grant 96-01-00947. 
Suppose now that we are given a function $\hat{u}: \mathbb{R} \rightarrow R$ and $\hat{x}>0$ such that the support of $\hat{u}$ is contained in $(-\infty,-\hat{x}]$ and let $\hat{y}: \bar{\Pi} \rightarrow \mathbb{R}$ be the solution to the equation:

$$
L \hat{y}=0,\left.\quad \hat{y}\right|_{\{0\} \times \mathbb{R}}=\hat{u} .
$$

Given positive bounds $0<x_{0}<x_{1}$, we seek a function $u$ with support in $\left[x_{0}, x_{1}\right]$ such that the corresponding solution $y$ satisfies:

$$
y(t, 0)=\hat{y}(t, 0) \quad \forall t \in[0, T]
$$

This is an ill-posed linear problem. We prove in Section 3 of this article that, under some regularity conditions on the coefficients $a_{i}(t, x)$, if a solution exists in the space of continuous functions with polynomial growth at infinity, it is unique in this space. Then we show in Section 4 a density theorem, namely, for any $\varepsilon>0$, there is a continuous function $u$ with support in $\left[x_{0}, x_{1}\right]$ such that:

$$
\max _{0 \leq t \leq T}|y(t, 0)-\hat{y}(t, 0)| \leq \varepsilon
$$

The regularity conditions on coefficients are precisely stated in Section 2. To summarise, we require uniform parabolicity on the domain $\bar{\Pi}$, Hölder continuity of $a_{i}$ and its derivatives up to order $i\left(C^{1,2}\right.$ for $\left.a_{2}\right)$, analyticity in $x$ for small $t$ and $x>x_{1}$, and stabilisation from the right hand side for $a_{1}$ and $a_{2}$ (their derivatives should decrease with a power rate when $x$ tends to $+\infty)$.

A first version of this paper was available in 1998 as a preprint, see [3]. Some extensions of the results are due to Shorygin [24], who obtains explicit bounds for $\|\hat{u}\|_{L^{2}}$ with respect to $\|y(., 0)-\hat{y}(., 0)\|_{L^{2}}$.

\subsection{Financial origin of the question}

\subsubsection{Barrier options in the generalised Black-Scholes model}

This problem finds its origin in the hedging of a certain type of options - namely "barrier options" - in financial markets. A barrier option on an asset $S$ (a stock, an index, a currency, a commodity, etc.) with "pay-off" $\phi(S)$, maturity $M$ - a date posterior to the current date $t_{0}$ - and "up-and-out barrier" $H$ delivers an amount $\phi\left(S_{M}\right)$ of numeraire at maturity, if $S_{M}$ is the price of the underlying asset at this date, provided for any $t \in\left[t_{0}, M\right]$, the asset price $S_{t}$ did not cross the barrier, i.e.:

$$
S_{t}<H \quad \forall t \in\left[t_{0}, M\right]
$$

Similarly, there exist "down-and-out", "up-and-in" and "down-and-in" barrier options, as well as "double barrier" options. An option without barriers and no exercise possibility prior to the maturity date is called "European". The "pay-off function" $\phi(S)$ is typically a piecewise linear function:

$$
\begin{aligned}
& \phi(S)=\max (S-K, 0) \quad \text { "Call with strike } K " \\
& \phi(S)=\max (K-S, 0)
\end{aligned} \quad \text { "Put with strike } K " .
$$

In a generalised Black-Scholes setting the underlying price process $S_{t}$, which is assumed to be Markov, is a positive Ito process:

$$
\frac{\mathrm{d} S_{t}}{S_{t}}=\mu\left(t, S_{t}\right) \mathrm{d} t+\sigma\left(t, S_{t}\right) \mathrm{d} W
$$

See [4] and [21] for the original model with constant coefficients. The generalised framework, with coefficients depending on the time and on the underlying, is described in [12] and [8]. 
Under the assumption of "absence of arbitrage opportunity" (AAO), the option is exactly replicable with a dynamic hedging strategy and its price $P(t, S)$ satisfies the following parabolic equation, called Black-Scholes equation:

$$
\frac{\partial P}{\partial t}+\frac{1}{2} \sigma(t, S)^{2} S^{2} \frac{\partial^{2} P}{\partial S^{2}}+(r(t)-q(t)) S \frac{\partial P}{\partial S}-r(t) P=0 .
$$

In this equation, $r$ stands for the interest rate in the domestic currency, and $q$ is the dividend rate if the underlying is a stock or a stock index, the foreign interest rate if it is a currency or the "convenience yield" in the case of a commodity. It is widely admitted in financial models that they only depend on time $t$ - even if they are, sometimes, themselves stochastic processes - although our mathematical framework could allow them to be a function of both $t$ and $S$. Nevertheless, it is important that the volatility parameter $\sigma(t, S)$ be a function of both variables in order to be consistent with the market price of standard options (i.e. without barriers): this is the so-called "smile" effect.

Dirichlet-type boundary conditions for barrier options (which are obvious to translate in $(M-t, x)$ variables) are given on the rectangle $\tilde{\Pi}=\left[t_{0}, M\right] \times[0, H]$ by:

$$
\begin{cases}\left.P(t, S)\right|_{t=M}=\phi(S) & 0 \leq S \leq H \\ \left.P(t, S)\right|_{S=H}=0 & t_{0} \leq t \leq M\end{cases}
$$

and

$$
\left.P(t, S)\right|_{S=0}=\exp \left(-\int_{t}^{M} r(z) \mathrm{d} z\right) \phi(0) \quad t_{0} \leq t \leq M
$$

which is a consequence of the operator degeneracy on the axis $\{S=0\}$.

We get the shape (1.1) by setting $x=\log \frac{S}{H}$ and reverting the time $t \mapsto M-t, T=M-t_{0}$. With these changes of variables, one has:

$$
\begin{aligned}
& a_{2}(t, x)=\frac{1}{2} \sigma\left(M-t, H \mathrm{e}^{x}\right)^{2} \\
& a_{1}(t, x)=q(M-t)-r(M-t)+\frac{1}{2} \sigma\left(M-t, H \mathrm{e}^{x}\right)^{2} \\
& a_{0}(t, x)=r(M-t) .
\end{aligned}
$$

\subsubsection{Static hedging of barrier options}

The static hedging of barrier options was described in an article by Carr et al. [6] and in El Karoui [19]. In [6], it is assumed that the volatility $\sigma$ is constant and that $r=q=0$, in which case an explicit formula for the solutions of the parabolic equation with piecewise linear boundary conditions can be found (see [10] and [23]). The study in [19] is extended to non-zero $q$ and $r$, but still constant parameters. The idea of static hedging relies on the fact that European options - i.e. without barriers - are much more liquid and, in particular, traded at the Exchange, so that they can be used to hedge barrier options.

Suppose now that we have sold (resp. bought) an "up-and-out barrier" option with "barrier" $H$ and "payoff" $\hat{\phi}(S), S<H$. We let $P(t, S)$ be the price of the barrier option, that is, the solution to equation (1.2) with boundary conditions (1.3) in which $\phi(S)$ is replaced by $\hat{\phi}(S)$. We also let $\hat{Q}(t, S)$ be the price of the European option with "pay-off" $\hat{\phi}(S) \mathbf{1}_{\{S<H\}}$, that is, the same as the barrier option, but ignoring the barrier except at maturity. The function $\hat{Q}(t, S)$ is a solution of Black-Scholes equation (1.2) with only the first boundary conditions of (1.3):

$$
\left.\hat{Q}(t, S)\right|_{t=M}=\hat{\phi}(S) \mathbf{1}_{\{S<H\}} \quad S \in[0,+\infty) .
$$


Assume that we can find a "mirror" function $\phi(S)$ with support in $(H,+\infty)$, such that the solution $Q(t, S)$ to the Black-Scholes equation (1.2) with terminal condition:

$$
\begin{cases}\left.Q(t, S)\right|_{t=M}=\phi(S) \mathbf{1}_{\{S>H\}} & S \in[0,+\infty) \\ \left.Q(t, S)\right|_{S=0}=0 & t_{0} \leq t \leq M\end{cases}
$$

and no barrier assumptions, happens to coincide with $\hat{Q}(t, S)$ along the barrier $\left[t_{0}, M\right) \times\{H\}$ :

$$
Q(t, H)=\hat{Q}(t, H) \quad \forall t \in\left[t_{0}, M\right)
$$

Then the hedging strategy consists in buying (resp. selling) a combination of European options - calls and puts with maturity $M$ - that reproduce the "pay-off" function $\hat{\phi}(S) \mathbf{1}_{\{S<H\}}$, and selling (resp. buying) another combination of European options - calls and puts, again with maturity $M$ - that reproduce the "pay-off" function $\phi(S) \mathbf{1}_{\{S>H\}}$. The price of the overall combination is $\hat{Q}(t, S)-Q(t, S)$. It is a solution of equation (1.2) and happens to satisfy the same boundary conditions as $P(t, S)$ on $\partial \tilde{\Pi}$ :

$$
\left.(\hat{Q}(t, S)-Q(t, S))\right|_{S=H} \equiv 0 \quad t \in\left[t_{0}, M\right]
$$

therefore the two functions are equal. In other words, as long as the underlying price $S_{t}$ does not reach the barrier, the barrier option and the combination of European options exactly have the same price. As soon as the underlying reaches the level $S_{t}=H$, then the barrier option disappears but, at the same time, the combination of European options vanishes in price, i.e. one can use the exact amount received by selling options that were bought to buy back options that were sold. In the case where the maturity is reached while the underlying did not cross the barrier, then the "pay-off" of the combination of European options, i.e. $\hat{\phi}(S)$, exactly matches the amount due to the buyer (resp. expected from the seller) of the barrier option.

Note that the $C^{0}$-density theorem, together with the fact that any continuous function can be approximated by piecewise linear ones, prove that unwinding the option portfolio can be made at an arbitrary low cost, whatever the time the barrier is reached, if ever.

This is of course a theoretical framework where no margin ("transaction costs") are applied to the "buying" and "selling" prices of options. We also assume that the "volatility surface", that is the function $\sigma(t, S)$, is a fixed function which does not depend on the evaluation date $t_{0}$. In practice, one should constantly re-calibrate this function with respect to the current price of European options, so that it is not clear that a combination of options which, theoretically, would vanish in price as forecasted at time $t_{0}$ will indeed have price zero when the barrier is really crossed. The last remark is the time taken to "unwind" the portfolio in case of barrier crossing, which may imply some mismatch in the compensation of buys by sales (problem of "slippage"). For the reader who is interested in the practical management of options, one of the best references is Taleb [25].

Andersen et al. [1] provide a very practical method to numerically build static hedges of various kinds of barrier options. Unlike our approach, their hedges involve options with maturities ranging from $t_{0}$ to $M$, which, at least theoretically, implies a continuous management of maturing options.

Theoretical studies in more extended mathematical frameworks that involve market imperfections can be found in Avellaneda and Paras [2] and Davis et al. [7] for the problem of transaction costs and in Dupire [13] and Derman and Kani [9] for stochastic volatility surfaces. Dubourg's thesis [11] is fully devoted to market imperfections. The market slippage is due to the lack of liquidity. Finding models that properly measure its impact is difficult, but Taleb's book thoroughly study this issue, as this is one of the toughest practical difficulties of traders - and probably one of the major limitations to the applicability of static hedging. 


\section{Statement of the PRoblem}

As stated in the introduction, we are given a parabolic non-degenerate diffusion equation:

$$
L y=\partial_{t} y(t, x)-a_{2}(t, x) \partial_{x x} y(t, x)+a_{1}(t, x) \partial_{x} y(t, x)+a_{0}(t, x) y(t, x)=0 .
$$

The differential operator $L$ is defined on the domain $\Pi=(0, T) \times \mathbb{R}$. We assume that the coefficients $a_{0}(t, x)$, $a_{1}(t, x)$ and $a_{2}(t, x)$ satisfy the following conditions:

Condition 2.1 (parabolicity). The coefficients $a_{i}(t, x), i=0,1,2$ are real-valued functions defined on $\bar{\Pi}$ and there exists $\alpha_{1}>\alpha_{0}>0$ such that:

$$
\alpha_{0} \leq a_{2}(t, x) \leq \alpha_{1} \quad \forall(t, x) \in \bar{\Pi} .
$$

It is well know that, under very mild regularity assumptions on the coefficients and on the initial data $u(x)$ :

$$
\left.y(t, x)\right|_{t=0}=u(x)
$$

$\left(u(x)\right.$ should be continuous with growth at infinity at most like $\left.|x|^{m}, m>0\right)$ the problem (2.1) is well posed, i.e. it has a unique well defined solution in $\Pi$ which continuously depends on the boundary data.

At variance nothing similar is true for the problem described in Section 1.1. Let $\hat{x}, x_{1}$ and $x_{2}$ be given positive numbers such that:

$$
\hat{x}>0 \quad 0<x_{0}<x_{1} .
$$

Let $\hat{y}(t, x)$ be the solution of the Cauchy problem $(2.1,2.2)$ :

$$
L \hat{y}=0,\left.\quad \hat{y}\right|_{t=0}=\hat{u}
$$

with initial data $u(x) \equiv \hat{u}(x)$ concentrated in the set $(-\infty,-\hat{x}]$ :

$$
\operatorname{supp} \hat{u} \subset(-\infty,-\hat{x}] \text {. }
$$

The question is to find an initial condition $u(x) \in C^{0}(\mathbb{R})$ concentrated in $\left[x_{0}, x_{1}\right]$ :

$$
\operatorname{supp} u \subset\left[x_{0}, x_{1}\right]
$$

such that the solution $y(t, x)$ of the Cauchy problem $(2.1,2.2)$ with initial condition $u(x)$ satisfies the equality:

$$
\left.y(t, x)\right|_{x=0}=\left.\hat{y}(t, x)\right|_{x=0} .
$$

If such a function $u$ does not exist then one may ask whether it is possible to find a sequence $u_{n}(x) \in$ $C(\mathbb{R})$, supp $u_{n} \subset\left[x_{0}, x_{1}\right]$ such that the sequence of solutions $y_{n}(t, x)$ of $(2.1,2.2)$ with initial value $u=u_{n}$ satisfies the relation:

$$
\lim _{n \rightarrow+\infty} \sup _{0 \leq t \leq T}\left|y_{n}(t, 0)-\hat{y}(t, 0)\right|=0 .
$$

Since this problem is ill-posed, as it will appear below (in particular a solution exists only for a dense set of initial data $\hat{u}$ ) more regularity than for the direct problem is needed on the coefficients. In the whole sequel, we assume that the following "technical" conditions are fulfilled. 
Condition 2.2 (smoothness). The functions $a_{i}(t, x), i=0,1,2$ are continuous and bounded on $\bar{\Pi}, a_{1} \in C^{1}(\bar{\Pi})$ and $a_{2}(t, x) \in C^{1,2}(\bar{\Pi})$. Furthermore there are two constants $A>0$ and $0<\gamma<1$ such that for any $(t, x) \in \bar{\Pi}$ and $\left(t_{0}, x_{0}\right) \in \bar{\Pi}$ the following Hölder condition is true:

$$
\left|\frac{\partial^{k} a_{i}(t, x)}{\partial x^{k}}-\frac{\partial^{k} a_{i}\left(t_{0}, x_{0}\right)}{\partial x^{k}}\right| \leq A\left(\left|x-x_{0}\right|^{\gamma}+\left|t-t_{0}\right|^{\gamma}\right)
$$

where $0 \leq i \leq 2,0 \leq k \leq i$ with the convention $\partial^{0} a(x) / \partial x^{0} \equiv a(x)$.

Condition 2.3 (analyticity). There exists $\delta_{0}>0$ such that for any $t \in\left[0, \delta_{0}\right]$ the functions $a_{i}(t, x), i=0$, 1,2 are analytic with respect to $x$ on $\left[x_{1},+\infty\right)$.

Condition 2.4 (stabilization from the right hand side). The following inequalities are true as $x \longrightarrow+\infty$ :

$$
\left\{\begin{array}{l}
\left|\partial_{t} a_{2}(t, x)\right| \leq c x^{-\alpha} \\
\left|\partial_{x} a_{2}(t, x)\right| \leq c x^{-\alpha-1} \\
\left|\partial_{t} a_{1}(t, x)\right|+\left|\partial_{x} a_{1}(t, x)\right| \leq c x^{-\alpha}
\end{array}\right.
$$

where $c$ and $\alpha$ are strictly positive constants that are independent of $t$.

\section{The Uniqueness THEOREM}

\subsection{Uniqueness}

For any solution $\hat{y}(t, x)$ of $(2.1)$ and $(2.2)$ with $\hat{u} \in C^{0}(\mathbb{R})$, supp $\hat{u} \subset(-\infty, \hat{x}]$, we show here that there is at most one initial data $u \in C^{0}(\mathbb{R})$ satisfying:

$$
\left\{\begin{array}{l}
|u(x)| \leq C x^{m}, \quad m>0 \\
\operatorname{supp} u \subset\left[x_{0},+\infty\right), \quad x_{0}>0
\end{array}\right.
$$

where $C$ is a positive constant, and such that the corresponding solution $y(t, x)$ coincides with $\hat{y}(t, x)$ on $\{(t, x) \in \Pi, x=0\}$. Observe that the uniqueness theorem holds in a slightly broader class than expected. It is not assumed that supp $u$ is bounded, however, $u$ must mildly increase for $x$ going to $+\infty$.

The problem being linear, it suffices to show that if a continuous initial condition $u$ satisfies (3.1) and if the solution $y(t, x)$ of equations $(2.1,2.2)$ vanishes for $x=0$, then $u=0$.

Theorem 3.1. Let $u(x) \in C^{0}(\mathbb{R})$ satisfy (3.1). If the solution $y(t, x)$ of equations (2.1, 2.2) satisfies:

$$
\forall t \in(0, T) \quad y(t, 0)=0
$$

then one must have:

$$
\forall x \in \mathbb{R} \quad u(x)=0
$$

Proof. The function $y(t, x)$ satisfies the equation:

$$
\partial_{t} y(t, x)-a_{2} \partial_{x x} y(t, x)+a_{1} \partial_{x} y(t, x)+a_{0} y(t, x)=0
$$

for $(t, x) \in \Pi_{-}=(0, T) \times(-\infty, 0)$, as well as the initial and boundary conditions:

$$
\begin{aligned}
y(0, x)=0, & \forall x<0 \\
y(t, 0)=0, & \forall t \in(0, T) .
\end{aligned}
$$


Therefore its restriction to $\Pi_{-}$is a solution of a well-posed Dirichlet problem with zero initial and boundary (for $x=0$ ) data. This implies:

$$
\forall(t, x) \in \Pi_{-} \quad y(t, x) \equiv 0 .
$$

Hence on the line $\{(t, x) \in \Pi, x=0\}$, one has:

$$
\left\{\begin{array}{c}
\left.y(t, x)\right|_{x=0}=0 \\
\left.\partial_{x} y(t, x)\right|_{x=0}=0 .
\end{array}\right.
$$

As $y(t, x)$ also satisfies $(3.3)$ for $(t, x) \in \Pi_{+} \equiv(0, T) \times(0, \infty)$, the standard Holmgren theorem for parabolic equations (see for instance Tataru [26] for recent proofs and references) implies, thanks to the mild growth assumption at infinity for $u$, that $y(t, x)$ again vanishes in $\Pi_{+}$. In particular:

$$
\forall x \in \mathbb{R} \quad u(x)=\left.y(t, x)\right|_{t=0} \equiv 0 .
$$

\subsection{Non existence results}

The uniqueness theorem leads to a non existence result.

Corollary 3.2. For a wide class of parabolic equations problem (2.1, 2.2, 2.6) does not possess a solution $u(x)$ in the class of $u(x) \in C^{0}(\mathbb{R})$ satisfying condition (2.5).

Proof. The first type of obstruction is based on the support of $u$. Suppose that the coefficients $a_{0}(t, x)$ and $a_{2}(t, x)$ of equation (2.1) are even functions with respect to $x$, and that $a_{1}(t, x)$ is an odd function:

$$
a_{i}(t, x)=(-1)^{i} a_{i}(t,-x) \quad i=0,1,2
$$

We assume that the support of $\hat{u}(x)$ coincides with the interval $\left[-\hat{x}_{1},-\hat{x}\right]$, where $-\infty \leq-\hat{x}_{1}$. Equalities $(3.8)$ imply that $\hat{y}(t,-x)$ is also a solution of (2.1). Therefore, the initial condition $\hat{u}(-x)$ is the solution of problem $(2.1,2.2,2.7)$. This solution belongs to the class defined by (3.1) and (3.2). From Theorem 3.1, it is unique in this class, therefore one has:

$$
\left[\hat{x}, \hat{x}_{1}\right] \subseteq\left[x_{0}, x_{1}\right]
$$

If this inclusion is not satisfied, then there is no solution with support in $\left[x_{0}, x_{1}\right]$.

The support of $u$ is not the only obstacle to the existence of solutions. It is indeed possible to build an example for which no solution with a support contained in the whole interval $[0, \infty$ [ will exist. Let us introduce the diffusion equation:

$$
L y=\partial_{t} y-\partial_{x x} y+b(t) \partial_{x} y .
$$

The function $b(t)$ is assumed to be smooth and nonnegative, equal to 0 for $0 \leq t \leq T_{1}$ and strictly positive for $T_{1}<t \leq T$. Denote by $B(t)$ the function:

$$
B(t)=\int_{0}^{t} b(s) \mathrm{d} s .
$$

The solution $y(t, x)$ to the evolution equation:

$$
L y=0, \quad y(0, x)=u(x)
$$


can be explicitly computed by Fourier transform and is given by the formula:

$$
y(x, t)=\frac{1}{\sqrt{4 \pi t}} \int_{-\infty}^{+\infty} \exp \left(-\frac{|x-\xi+B(t)|^{2}}{4 t}\right) u(\xi) \mathrm{d} \xi .
$$

We start with a nonnegative initial data $\hat{u}(x)$ with support in $(-\infty, 0]$. From the first example, and from the fact that $B$ vanishes on $\left[0, T_{1}\right]$, the solution $u$ (provided it exists) must satisfy the relation:

$$
\hat{u}(x)=u(-x) .
$$

For $t \geq T_{1}$ the difference between the two solutions on the line $\{(t, x) \in \Pi, x=0\}$ is be given by:

$$
\frac{1}{\sqrt{4 \pi t}} \int_{-\infty}^{0}\left\{\exp \left(-\frac{|\xi-B(t)|^{2}}{4 t}\right)-\exp \left(\frac{|\xi+B(t)|^{2}}{4 t}\right)\right\} \hat{u}(\xi) \mathrm{d} \xi
$$

which is nonzero for a general $\hat{u}$.

Remark 1. Carr and Chou [5] provide an exact solution for $u$ with various pay-off functions $\hat{u}$ in the case of a constant $b$. From these examples, it can be seen that, except very particular shapes of $\hat{u}$, the solution $u$ is, in general, supported by the whole half-line $(-\infty, 0]$ and that different values of $b$ lead to different solutions, which cannot be merged to produce a solution to, for instance, the case where $b(t)$ is a "bump" function, as described above, or even a step function.

These negative answers to the first question stated in the end of Section 2 justify the seek for approximate solutions. This is the purpose of the next section.

\section{Approximate solutions}

In this section we prove the existence of a sequence $u_{n}(x)$ of initial conditions satisfying (2.5), such that the corresponding solutions $y_{n}(t, x)$ of $(2.1,2.2)$ uniformly converge to $\hat{y}$ on the line $\{x=0\}$ :

$$
\lim _{n \rightarrow+\infty} \sup _{0 \leq t \leq T}\left|\hat{y}(t, 0)-y_{n}(t, 0)\right|=0 .
$$

The proof relies both on a duality argument and on the uniqueness property. This method was used already in many situations (see for instance J.-L. Lions and Lattès [20], J.-L. Lions [22], Fursikov [15], Fursikov and Imanuvilov [16]). As we are willing $C^{0}$-convergence - which is the only meaningful convergence for the financial problem -, the duality argument will involve solutions of the diffusion equation with a measure on the right hand side.

Theorem 4.1. Suppose that $\hat{u}(x) \in C^{0}(\mathbb{R})$ satisfies (2.4) and that $\hat{y}(t, x)$ is the solution of problem (2.3), where $L$ is the operator defined in (2.1). Let $0<x_{0}<x_{1}<\infty$ be given. There exists a sequence of functions $u_{n}(x) \in C^{0}(\mathbb{R})$ satisfying (2.5), such that the solutions $y_{n}(t, x)$ of (2.1,2.2) with $u(x) \equiv u_{n}(x)$ satisfy (4.1).

Remark 2. If, as in the examples above, an exact solution $u$ does not exist, then the sequence $u_{n}$ cannot converge. In particular, it will not be bounded for any norm which is relatively compact with respect to $C^{0}$, or to any functional space in which the uniqueness theorem is valid.

Remark 3. In practice, shrinking the support may have a dramatic impact on the size of the solution. The figure below shows the function $u$ that minimises $\|\hat{y}(., 0)-y(., 0)\|_{L^{2}}$ when $L$ is given by (3.9) with $b=0$, $x_{0}=0.1, x_{1}=2, \hat{x}=-0.2$ and $\hat{u}(x)=\hat{x}-x$ for $x \in(-\infty, \hat{x}]$. 
Proof. Let $W$ be the subspace of traces $w(t) \equiv y(t, 0)$ of functions $y(t, x)$ satisfying $(2.1,2.2)$ for initial data $u(x)$ in the set

$$
U=\left\{u \in C^{0}(\mathbb{R}), \quad \operatorname{supp} u \subset\left[x_{0}, x_{1}\right]\right\} .
$$

If $W$ is not dense in $C^{0}(0, T)$, then there exists a nonzero measure $h(\mathrm{~d} t)$, such that

$$
\int_{0}^{T} w(t) h(\mathrm{~d} t)=0
$$

for any $w \in W$. Introduce the solution $p(t, x)$ of the following adjoint problem:

$$
\begin{gathered}
\partial_{t} p(t, x)+\partial_{x x}\left(a_{2}(t, x) p(t, x)\right)+\partial_{x}\left(a_{1}(t, x) p(t, x)\right)-a_{0}(t, x) p(t, x)=\delta(x) h(\mathrm{~d} t) \\
\left.p(t, x)\right|_{t=T}=0
\end{gathered}
$$

where $\delta(x)$ is the Dirac $\delta$-function. With the change of time variable $t \rightarrow T-t(4.4,4.5)$ turns out to be a classical well posed parabolic problem (see Friedman [11]). Multiplication of both sides of (4.4) by any solution $y(t, x)$ of $(2.1,2.2)$ followed by integration over $\Pi$ and integration by part (all these operations being done in the sense of distributions) leads to the formula:

$$
\begin{aligned}
0 & =\int_{\Pi}\left(\partial_{t} p+\partial_{x x}\left(a_{2} p\right)+\partial_{x}\left(a_{1} p\right)-a_{0} p\right) y \mathrm{~d} x \mathrm{~d} t-\int_{0}^{T} y(0, t) h(\mathrm{~d} t) \\
& =-\int_{x_{0}}^{x_{1}} p(0, x) u(x) \mathrm{d} x-\int_{\Pi}\left(\partial_{t} y-a_{2} \partial_{x x} y+a_{1} \partial_{x} y+a_{0} y\right) p \mathrm{~d} x \mathrm{~d} t \\
& =-\int_{x_{0}}^{x_{1}} p(0, x) u(x) \mathrm{d} x
\end{aligned}
$$

Since $u(x)$ runs through the whole space $U$, which can be identified to $C_{0}^{0}\left(\left[x_{0}, x_{1}\right]\right)=\left\{u \in C^{0}(\mathbb{R}), u\left(x_{0}\right)=\right.$ $\left.u\left(x_{1}\right)=0\right\}$ equality (4.6) implies that:

$$
p(0, x) \equiv 0, \quad \forall x \in\left[x_{0}, x_{1}\right]
$$

Since the coefficients $a_{i}$ of (4.4) satisfy condition 2.3 (of analyticity), the solution $p(t, x)$ to the parabolic equation (4.4) is an analytic function with respect to the variable $x$ for $t=0$ and $x \in\left[x_{1}, \infty\right)$, and the relation (4.7) implies the identity:

$$
p(0, x) \equiv 0, \quad \forall x \geq x_{0}
$$

The following assertion which will be proved in the next section, is the main step in the proof of Theorem 4.1.

Lemma 4.1. Let $p(t, x)$ satisfy equation (4.4) for $(t, x) \in \Pi_{N} \equiv(0, T) \times(N, \infty)$, with $N>0$ large enough, assume that the relations:

$$
\left.p(t, x)\right|_{t=0}=\left.p(t, x)\right|_{t=T}=0, \quad x>N
$$

hold and suppose that there exists a constant $\beta>0$, such that the function $r(t, x)=p(t, x) \mathrm{e}^{\beta x^{2}}$ belongs to the class:

$$
H^{1,2}\left(\Pi_{N}\right)=\left\{z(t, x) \in L^{2}\left([0, T] ; H^{2}(N, \infty)\right): \partial_{t} z \in L^{2}\left(\Pi_{N}\right)\right\} .
$$

Then $p(t, x)$ is identically zero in $\Pi_{N+1}$.

Continuation of the proof of Theorem 4.1. First observe that for any $N \geq x_{1}$ the solution $p(t, x)$ of problem $(4.4,4.5)$ satisfies the assumptions of Lemma 4.1. With $N \geq x_{1}$ (4.9) follows from (4.5, 4.8). To check the regularity and the growth condition (4.10), which will be used in conjunction with Carleman estimate in the Section 5 , one introduces the Green function $\Gamma(\tau, \xi, t, x)$ of the operator defined by the left hand side of (4.4). 
It is well-known (see Friedman [14]) that the Green function $\Gamma$ and its derivatives satisfy for $\tau>t$ the following estimates:

$$
\begin{aligned}
|\Gamma(\tau, \xi, t, x)| & \leq c(\tau-t)^{-\frac{1}{2}} \exp \left(-\frac{\lambda|x-\xi|^{2}}{(\tau-t)}\right) \\
\left|\partial_{x} \Gamma(\tau, \xi, t, x)\right| & \leq c(\tau-t)^{-\frac{3}{2}} \exp \left(-\frac{\lambda|x-\xi|^{2}}{(\tau-t)}\right) \\
\left|\partial_{t} \Gamma(\tau, \xi, t, x)\right|+\left|\partial_{x x} \Gamma(\tau, \xi, t, x)\right| & \leq c(\tau-t)^{-\frac{5}{2}} \exp \left(-\frac{\lambda|x-\xi|^{2}}{(\tau-t)}\right)
\end{aligned}
$$

where $c$ and $\lambda$ are positive constants. The solution $p$ of the problem $(4.4,4.5)$ is expressed, with respect to the Green function, as follows:

$$
p(t, x)=\int_{t}^{T} \Gamma(\tau, 0, t, x) h(\mathrm{~d} \tau) .
$$

This expression implies a uniform $C^{2}$ regularity on any domain of the form:

$$
\Delta_{k, K}=\{x, k \leq|x| \leq K\} \times[0, T], \quad 0<k<K<N .
$$

We now introduce a smooth truncation function $\theta(x)$ such that:

$$
\left\{\begin{array}{ccc}
\theta(x)=0 & \text { if } & |x| \leq k \\
\theta(x)=1 & \text { if } & |x| \geq K
\end{array}\right.
$$

and we set:

$$
\tilde{p}(t, x)=\theta(x) p(t, x) .
$$

The function $\tilde{p}(x, t)$ coincides with $p(x, t)$ for $|x| \geq K$ and is a solution of the following equation:

$$
\begin{gathered}
\partial_{t} \tilde{p}(t, x)+\partial_{x x}\left(a_{2}(t, x) \tilde{p}(t, x)\right)+\partial_{x}\left(a_{1}(t, x) \tilde{p}(t, x)\right)-a_{0}(t, x) \tilde{p}(t, x)=g(t, x) \\
\left.\tilde{p}(t, x)\right|_{t=T}=0
\end{gathered}
$$

where $g(t, x)$ denotes a $C^{2}$ function with support in the set $\Delta_{k, K}$, with $0<k<K<N$. Therefore, for $|x| \geq N$, one has:

$$
p(x, t)=\int_{-\infty}^{\infty} \int_{t}^{T} \Gamma(\tau, \xi, t, x) g(\tau, \xi) \mathrm{d} \tau \mathrm{d} \xi .
$$

Together with (4.11), this formula implies, for $|x|>N$, the raw estimate:

$$
\begin{aligned}
|p(x, t)| & \leq C \int_{-\infty}^{\infty} \int_{t}^{T}(\tau-t)^{-\frac{1}{2}} \exp \left(-\frac{\lambda|| x|-\xi|^{2}}{\tau-t}\right)|g(\tau, \xi)| \mathrm{d} \tau \mathrm{d} \xi \\
& \leq C K \exp \left(-\frac{\lambda|| x|-K|^{2}}{\tau-t}\right) \int_{t}^{T}(\tau-t)^{-\frac{1}{2}} \mathrm{~d} \tau .
\end{aligned}
$$

Estimates for the derivatives of $p$ can be obtained along the same line with a minor improvement of (4.18). Therefore all assumptions of the Lemma 4.1 are verified and, in virtue of this lemma, $p(t, x) \equiv 0$ for any $(t, x) \in \Pi_{N+1}$. The Holmgren theorem applied to the parabolic equation (4.4) implies that

$$
p(t, x)=0 \quad \forall(t, x) \in(0, T) \times(0, \infty) .
$$


In fact this property can be extended by continuity up to $x=0$ as follows. Thanks to (4.14) and Fubini's theorem, for any $\phi(t) \in C^{0}([0, T])$ the function $\Phi(x)=\int_{0}^{T} p(t, x) \phi(t) \mathrm{d} t$ is given by the formula:

$$
\begin{aligned}
\Phi(x) & =\int_{0}^{T} \phi(t) \int_{t}^{T} \Gamma(\tau, 0, t, x) h(\mathrm{~d} \tau) \mathrm{d} t \\
& =\int_{0}^{T}\left(\int_{0}^{\tau} \phi(t) \Gamma(\tau, 0, t, x) \mathrm{d} t\right) h(\mathrm{~d} \tau) .
\end{aligned}
$$

From (4.11) and thanks to the upper bound:

$$
\left|\int_{0}^{\tau} \phi(t) \Gamma(\tau, 0, t, x) \mathrm{d} t\right| \leq C \int_{0}^{\tau} \frac{|\phi(t)|}{\sqrt{\tau-t}} \mathrm{~d} t \leq \mathrm{cst}
$$

we may apply Lebesgue's convergence theorem as soon as:

$$
\forall t \in(0, T) \quad \lim _{x \rightarrow 0} \Gamma(\tau, 0, t, x)=\Gamma(\tau, 0, t, 0) .
$$

In fact, we know that the Green function $\Gamma(\tau, \xi, t, x)$ is regular for $t /=\tau$ (see Taylor [27], Vol. 2, 7.13, and Friedman [14]). Eventually, it has been shown that $\Phi(x)$ is continuous in a neighbourhood of zero. Thanks to (4.19) one has:

$$
\lim _{x \rightarrow 0} \Phi(x)=\lim _{x \rightarrow 0^{+}} \int_{0}^{T} p(t, x) \phi(t) \mathrm{d} t=0 .
$$

Consequently:

$$
\left.p(t, x)\right|_{x=0}=0 .
$$

On $\Pi_{-}=(0, T) \times(-\infty, 0]$ the function $p(t, x)$ is the solution of a well-posed Dirichlet problem with initial and boundary data equal to zero and one has:

$$
p(t, x) \equiv 0 \quad \forall(t, x) \in(0, T) \times(-\infty, 0] .
$$

Equalities (4.19, 4.24) and equation (4.4) imply that $h(t) \equiv 0$.

\section{Carleman estimate}

This section is devoted to the proof of Lemma 4.1. To this end a Carleman type estimate is proved for a functions $p$ satisfying assumptions of Lemma 4.1. Note that estimates of such type were used for investigations of Cauchy problems (see for example Hörmander [18]) or controllability problems (see Fursikov and Imanuilov [17]), as well as in other areas. Let us introduce the weight function

$$
\varphi(x)=x^{2-\gamma}-(N+1)^{2-\gamma}
$$

where $\gamma$ is such that:

$$
0<\gamma<\min \left(\frac{1}{2}, \alpha\right) .
$$

In the above formula $\alpha$ is the number given by the condition 2.4 (stabilization). 
Lemma 5.1. Let $p(t, x)$ satisfy assumptions of Lemma 4.1. Then there exists a constant $c>0$ such that for any sufficiently large $s>0$ and $N>0$ the following inequality holds:

$$
\int_{\Pi_{N}}\left(s x^{-\gamma}\left(\partial_{x} p\right)^{2}+s^{3} x^{2-3 \gamma} p^{2}\right) \mathrm{e}^{2 s \varphi(x)} \mathrm{d} x \mathrm{~d} t \leq\left. c N^{3-3 \gamma} s^{3} \mathrm{e}^{-2 s N^{1-\gamma}} \int_{0}^{T}\left[\left(\partial_{t} p\right)^{2}+\left(\partial_{x} p\right)^{2}+p^{2}\right]\right|_{x=N} \mathrm{~d} t
$$

where $\varphi(x)$ is given by (5.1).

Proof. Introduce in equation (4.4) the function $q(t, x)$ given by:

$$
p(t, x)=\mathrm{e}^{-s \varphi(x)} q(t, x)
$$

This function satisfies:

$$
L_{1} q+L_{2} q=f_{s}
$$

where:

$$
\begin{aligned}
L_{1} q & =\partial_{t} q+\left(a_{1}-2 s a_{2} \partial_{x} \varphi\right) \partial_{x} q \\
L_{2} q & =a_{2} \partial_{x x} q+\left(s^{2} a_{2}\left(\partial_{x} \varphi\right)^{2}-s a_{1} \partial_{x} \varphi\right) q \\
f_{s} & =\left(s \partial_{x x} \varphi+2 s\left(\partial_{x} a_{2}\right) \partial_{x} \varphi-\partial_{x x} a_{2}-\partial_{x} a_{1}+a_{0}\right) q-2\left(\partial_{x} a_{2}\right) \partial_{x} q .
\end{aligned}
$$

Since $p$ satisfies (4.10), the relations (5.1) and (5.3) imply that $q \in H^{1,2}\left(\Pi_{N}\right)$. This inclusion justifies all transformations and integrations on $\Pi_{N}$ made below, starting from the formula:

$$
\left\|L_{1} q\right\|_{L^{2}\left(\Pi_{N}\right)}^{2}+\left\|L_{2} q\right\|_{L^{2}\left(\Pi_{N}\right)}^{2}+2\left(L_{1} q, L_{2} q\right)_{L^{2}\left(\Pi_{N}\right)}=\left\|f_{s}\right\|_{L^{2}\left(\Pi_{N}\right)}^{2} .
$$

With (5.5) and (5.6) one obtains as last term of the left hand side of (5.8):

$$
2\left(L_{1} q, L_{2} q\right)_{L^{2}\left(\Pi_{N}\right)}=I_{1}+I_{2}+I_{3}
$$

with

$$
\begin{aligned}
& I_{1}=2 \int_{\Pi_{N}}\left(\partial_{t} q\right)\left(a_{2} \partial_{x x} q+\left\{s^{2} a_{2}\left(\partial_{x} \varphi\right)^{2}-s a_{1} \partial_{x} \varphi\right\} q\right) \mathrm{d} x \mathrm{~d} t \\
& I_{2}=2 \int_{\Pi_{N}}\left(a_{1}-2 s a_{2} \partial_{x} \varphi\right)\left(\partial_{x} q\right) a_{2} \partial_{x x} q \mathrm{~d} x \mathrm{~d} t \\
& I_{3}=2 \int_{\Pi_{N}}\left(a_{1}-2 s a_{2} \partial_{x} \varphi\right)\left(\partial_{x} q\right)\left(s^{2} a_{2}\left(\partial_{x} \varphi\right)^{2}-s a_{1} \partial_{x} \varphi\right) q \mathrm{~d} x \mathrm{~d} t
\end{aligned}
$$

Integrations by parts give:

$$
\begin{aligned}
I_{1}= & -\left.2 \int_{0}^{T}\left(a_{2}\left(\partial_{t} q\right)\left(\partial_{x} q\right)\right)\right|_{x=N} \mathrm{~d} t \\
& +\int_{\Pi_{N}}\left\{-a_{2} \partial_{t}\left(\partial_{x} q\right)^{2}-2\left(\partial_{x} a_{2}\right)\left(\partial_{t} q\right)\left(\partial_{x} q\right)+\left(s^{2} a_{2}\left(\partial_{x} \varphi\right)^{2}-s a_{1} \partial_{x} \varphi\right) \partial_{t} q^{2}\right\} \mathrm{d} x \mathrm{~d} t \\
= & -\left.2 \int_{0}^{T}\left(a_{2}\left(\partial_{t} q\right)\left(\partial_{x} q\right)\right)\right|_{x=N} \mathrm{~d} t \\
& +\int_{\Pi_{N}}\left\{\left(\partial_{t} a_{2}\right)\left(\partial_{x} q\right)^{2}-2\left(\partial_{x} a_{2}\right)\left(\partial_{t} q\right)\left(\partial_{x} q\right)-\left(s^{2}\left(\partial_{t} a_{2}\right)\left(\partial_{x} \varphi\right)^{2}-s\left(\partial_{t} a_{1}\right) \partial_{x} \varphi\right) q^{2}\right\} \mathrm{d} x \mathrm{~d} t
\end{aligned}
$$




$$
\begin{aligned}
I_{2}= & \int_{\Pi_{N}}\left(a_{1} a_{2}-2 s a_{2}^{2} \partial_{x} \varphi\right) \partial_{x}\left(\partial_{x} q\right)^{2} \mathrm{~d} x \mathrm{~d} t \\
= & -\left.\int_{0}^{T}\left(\left(a_{1} a_{2}-2 s a_{2}^{2} \partial_{x} \varphi\right)\left(\partial_{x} q\right)^{2}\right)\right|_{x=N} \mathrm{~d} t \\
& \left.\quad+\int_{\Pi_{N}}\left\{2 s a_{2}^{2} \partial_{x x} \varphi+4 s a_{2}\left(\partial_{x} a_{2}\right) \partial_{x} \varphi-\partial_{x}\left(a_{1} a_{2}\right)\right\}\left(\partial_{x} q\right)^{2}\right\} \mathrm{d} x \mathrm{~d} t \\
I_{3}= & \int_{\Pi_{N}}\left\{-s a_{1}^{2} \partial_{x} \varphi+3 s^{2} a_{1} a_{2}\left(\partial_{x} \varphi\right)^{2}-2 s^{3} a_{2}^{2}\left(\partial_{x} \varphi\right)^{3}\right\} \partial_{x} q^{2} \mathrm{~d} x \mathrm{~d} t \\
= & \left.\int_{0}^{T}\left(\left(2 s^{3} a_{2}^{2}\left(\partial_{x} \varphi\right)^{3}-3 s^{2} a_{1} a_{2}\left(\partial_{x} \varphi\right)^{2}+s a_{1}^{2} \partial_{x} \varphi\right) q^{2}\right)\right|_{x=N} \mathrm{~d} t \\
& +\int_{\Pi_{N}}\left\{6 s^{3} a_{2}^{2}\left(\partial_{x} \varphi\right)^{2} \partial_{x x} \varphi+4 s^{3} a_{2}\left(\partial_{x} a_{2}\right)\left(\partial_{x} \varphi\right)^{3}-3 s^{2}\left(\partial_{x}\left(a_{1} a_{2}\right)\right)\left(\partial_{x} \varphi\right)^{2}\right. \\
& \left.-6 s^{2} a_{1} a_{2} \partial_{x} \varphi \partial_{x x} \varphi+2 s a_{1} \partial_{x} a_{1} \partial_{x} \varphi+s a_{1}^{2} \partial_{x x} \varphi\right\} q^{2} \mathrm{~d} x \mathrm{~d} t .
\end{aligned}
$$

From (5.1), we have:

$$
\left\{\begin{array}{l}
\partial_{x} \varphi=(2-\gamma) x^{1-\gamma} \\
\partial_{x x} \varphi=(2-\gamma)(1-\gamma) x^{-\gamma}
\end{array}\right.
$$

The Condition 2.4 on coefficients $a_{i}$ (stabilization) provides an estimate of the function $f_{s}$ given by (5.7) for $N$ large enough:

$$
\left\|f_{s}\right\|_{L_{2}\left(\Pi_{N}\right)}^{2} \leq \int_{\Pi_{N}}\left\{\left(c_{1} x^{-2 \gamma} s^{2}+c_{2}\right) q^{2}+c x^{-2(1+\alpha)}\left(\partial_{x} q\right)^{2}\right\} \mathrm{d} x \mathrm{~d} t .
$$

With the same arguments, lower bounds for the left hand side of (5.14) and (5.15) are obtained:

$$
\begin{gathered}
I_{2} \geq \int_{\Pi_{N}} 2 s a_{2}^{2}(2-\gamma)(1-\gamma) x^{-\gamma}\left(\partial_{x} q\right)^{2} \mathrm{~d} x \mathrm{~d} t \\
-c_{3} \int_{\Pi_{N}}\left(s x^{-\gamma-\alpha}+x^{-\alpha}\right)\left(\partial_{x} q\right)^{2} \mathrm{~d} x \mathrm{~d} t \\
-c_{4}\left(1+N^{1-\gamma} s\right) \int_{0}^{T}\left(\left.\partial_{x} q\right|_{x=N}\right)^{2} \mathrm{~d} t \\
I_{3} \geq \int_{\Pi_{N}} 6 a_{2}^{2}(2-\gamma)^{3}(1-\gamma) s^{3} x^{2-3 \gamma} q^{2} \mathrm{~d} x \mathrm{~d} t \\
-c_{5} \int_{\Pi_{N}}\left|s^{3} x^{2-3 \gamma-\alpha}+s^{2}\left(x^{2-2 \gamma-\alpha}+x^{1-2 \gamma}\right)+s x^{-\gamma}\right| q^{2} \mathrm{~d} x \mathrm{~d} t \\
-c_{6}\left(s^{3} N^{3-3 \gamma}+s^{2} N^{2-2 \gamma}+s N^{1-\gamma}\right) \int_{0}^{T} q^{2}(t, N) \mathrm{d} t .
\end{gathered}
$$

The function $L_{1} q$ given by (5.5) satisfies, for $x>N \gg 1$ :

$$
\begin{aligned}
\left|2\left(\partial_{x} a_{2}\right)\left(\partial_{t} q\right)\left(\partial_{x} q\right)\right| & \leq 2\left|\left(\partial_{x} a_{2}\right)\left(\partial_{x} q\right) L_{1} q\right|+2\left|\left(\partial_{x} a_{2}\right)\left(\partial_{x} q\right)^{2}\left(a_{1}-2 s a_{2} \partial_{x} \varphi\right)\right| \\
& \leq \frac{1}{2}\left|L_{1} q\right|^{2}+c_{7}\left|\partial_{x} q\right|^{2}\left(x^{-2-2 \alpha}+x^{-1-\alpha}+s x^{-\alpha-\gamma}\right)
\end{aligned}
$$


This relation is used to estimate $I_{1}$ for $N \gg 1$ as follows:

$$
\begin{aligned}
\left|I_{1}\right| \leq & c \int_{0}^{T}\left|\left(\partial_{t} q \partial_{x} q\right)\right|_{x=N} \mathrm{~d} t+\frac{1}{2} \int_{\Pi_{N}}\left(L_{1} q\right)^{2} \mathrm{~d} x \mathrm{~d} t \\
& +c_{8} \int_{\Pi_{N}}\left\{\left(\partial_{x} q\right)^{2}\left(x^{-\alpha}+s x^{-\alpha-\gamma}\right)+q^{2}\left(s^{2} x^{2-2 \gamma-\alpha}+s x^{1-\gamma}\right)\right\} \mathrm{d} x \mathrm{~d} t .
\end{aligned}
$$

From formula (5.1), one has:

$$
\begin{aligned}
\varphi(N) & =N^{2-\gamma}-(N+1)^{2-\gamma} \\
& =-N^{2-\gamma}\left(\left(1+\frac{1}{N}\right)^{2-\gamma}-1\right) \\
& \leq-N^{2-\gamma}\left(1+\frac{1}{N}-1\right)=-N^{1-\gamma} .
\end{aligned}
$$

With (5.3), we get in particular:

and

$$
\left\{\begin{array}{c}
|q(t, N)| \leq \mathrm{e}^{-s N^{1-\gamma}}|p(t, N)| \\
\left|\partial_{t} q(t, N)\right| \leq \mathrm{e}^{-s N^{1-\gamma}}\left|\partial_{t} p(t, N)\right|
\end{array}\right.
$$

$$
\left|\partial_{x} q(t, N)\right| \leq \mathrm{e}^{-s N^{1-\gamma}}\left(\left|\partial_{x} p(t, N)\right|+s N^{1-\gamma}|p(t, N)|\right) .
$$

Using $(5.18,5.19)$ and $(5.21-5.23)$, Condition 2.2 (of parabolicity) and the inequality $\gamma<\frac{2}{3}$, one estimates the scalar product (5.9) from below:

$$
\begin{aligned}
2\left(L_{1} q, L_{2} q\right)_{L_{2}\left(\Pi_{N}\right) \geq} & \int_{\Pi_{N}}\left\{\frac{8 s}{9} \alpha_{0}^{2} x^{-\gamma}\left(\partial_{x} q\right)^{2}+\frac{128}{27} \alpha_{0}^{2} s^{3} x^{2-3 \gamma} q^{2}\right\} \mathrm{d} x \mathrm{~d} t \\
& -c \int_{\Pi_{N}}\left\{\left(x^{-\alpha}+s x^{-\alpha-\gamma}\right)\left(\partial_{x} q\right)^{2}+\left(s^{3} x^{2-3 \gamma-\alpha}+s^{2} x^{2-2 \gamma-\alpha}+s x^{1-\gamma}\right) q^{2}\right\} \mathrm{d} x \mathrm{~d} t \\
& -\frac{1}{2} \int_{\Pi_{N}}\left(L_{1} q\right)^{2} \mathrm{~d} x \mathrm{~d} t-\left.c^{\prime} s^{3} N^{3-3 \gamma} \mathrm{e}^{-2 s N^{1-\gamma}} \int_{0}^{T}\left(|p|^{2}+\left|\partial_{t} p\right|^{2}+\left|\partial_{x} p\right|^{2}\right)\right|_{x=N} \mathrm{~d} t
\end{aligned}
$$

Substitution of (5.24) and (5.17) into (5.8), with simple transformations, yields:

$$
\begin{array}{r}
\frac{1}{2}\left\|L_{1} q\right\|_{L_{2}\left(\Pi_{N}\right)}^{2}+\left\|L_{2} q\right\|_{L_{2}\left(\Pi_{N}\right)}^{2}+\int_{\Pi_{N}}\left\{\frac{8 s}{9} \alpha_{0}^{2} x^{-\gamma}\left(\partial_{x} q\right)^{2}\right. \\
\left.\quad+\frac{128}{27} \alpha_{0}^{2} s^{3} x^{2-3 \gamma} q^{2}\right\} \mathrm{d} x \mathrm{~d} t \\
\leq\left. c^{\prime} s^{3} N^{3-3 \gamma} \mathrm{e}^{-2 s N^{1-\gamma}} \int_{0}^{T}\left(|p|^{2}+\left|\partial_{t} p\right|^{2}+\left|\partial_{x} p\right|^{2}\right)\right|_{x=N} \mathrm{~d} t+c^{\prime \prime} \int_{\Pi_{N}}\left(x^{-\alpha}+s x^{-\alpha-\gamma}\right)\left(\partial_{x} q\right)^{2} \mathrm{~d} x \mathrm{~d} t \\
\quad+\int_{\Pi_{N}}\left(s^{3} x^{2-3 \gamma-\alpha}+s^{2} x^{2-2 \gamma-\alpha}+s x^{1-\gamma}\right) q^{2} \mathrm{~d} x \mathrm{~d} t
\end{array}
$$

Inequality (5.25) implies for $N$ and $s$ large enough, the estimate:

$$
\int_{\Pi_{N}}\left(\frac{\alpha_{0}^{2} s}{2} x^{-\gamma}\left(\partial_{x} q\right)^{2}+\alpha_{0}^{2} s^{3} x^{2-3 \gamma} q^{2}\right) \mathrm{d} x \mathrm{~d} t \leq\left. c^{\prime} s^{3} N^{3-3 \gamma} \mathrm{e}^{-2 s N^{1-\gamma}} \int_{0}^{T}\left(|p|^{2}+\left|\partial_{t} p\right|^{2}+\left|\partial_{x} p\right|^{2}\right)\right|_{x=N} \mathrm{~d} t .
$$

We now get the required estimate (5.2) by substituting $q$ in the left hand side of (5.26) by its expression with respect to $p$ as in (5.3). 
Proof of Lemma 4.1. Inequality (5.2) implies that for $s$ and $N$ large enough one has:

$$
\left.\int_{\Pi_{N+1}}\left(s x^{-\gamma}\left(\partial_{x} p\right)^{2}+s^{3} x^{2-3 \gamma} p^{2}\right) \mathrm{e}^{2 s \varphi(x)} \mathrm{d} x \mathrm{~d} t c N^{3-3 \gamma} s^{3} \mathrm{e}^{-2 s N^{1-\gamma}} \int_{0}^{T}\left\{\left(\partial_{t} p\right)^{2}+\left(\partial_{x} p\right)^{2}+p^{2}\right\}\right|_{x=N} \mathrm{~d} t
$$

with a constant $c$ which does not depend on $s$. The right hand side of this letter estimate tends to zero nonincreasingly as $s \longrightarrow+\infty$. If the function $p(t, x)$ is not identically zero on $\Pi_{N+1}$, then the left hand side of this equation goes to $+\infty$ as $s \longrightarrow+\infty$, which leads to a contradiction.

\section{Conclusion}

We have proved that the principle of static hedging of barrier options can be extended to the case of arbitrary diffusion coefficients for the underlying - provided some technical conditions are satisfied - for $C^{0}$ approximations only, but not as an exact hedge in general. If it exists, we also showed that such a hedge is unique. The proof of the density theorem deeply uses Carleman estimates on solutions of parabolic equations.

This article addresses a new class of inverse problems, which deserves further investigations. In particular we are convinced that our $C^{0}$ framework is neither optimal for the uniqueness, nor for the approximation result. Shorygin [24] studied the existence of $L^{2}$ bounds for approximate solutions. Other approaches should also be considered. Another interesting issue is to let both $\hat{u}$ and $u$ have their support contain the origin and examine the local behaviour of $u$ near the origin with respect to that of $\hat{u}$ and of the coefficients $a_{i}$, including when $\hat{u}$ is discontinuous - as is the case for "reverse barrier options".

The authors wish to thank the referees for providing many interesting references in this topic.

\section{REFERENCES}

[1] L. Andersen, J. Andreasen and D. Eliezer, Static Replication of Barrier Options: Some General Results. Preprint Gen. Re Fin. Prod. (2000).

[2] M. Avellaneda and A. Paras, Managing the Volatility Risk of Portfolio of Derivative Securities: The Lagrangian Uncertain Volatility Model. Appl. Math. Finance 3 (1996) 21-52.

[3] C. Bardos, R. Douady and A. Fursikov, Static Hedging of Barrier Options with a Smile: An Inverse Problem, Preprint CMLA No. 9810. École Normale Supérieure de Cachan (1998).

[4] F. Black and M. Scholes, The Pricing of Options and Corporate Liabilities. J. Polit. Econ. 81 (1973) 637-654.

[5] P. Carr and A. Chou, Breaking Barriers. RISK (1997) 139-145.

[6] P. Carr, K. Ellis and V. Gupta, Static Hedging of Exotic Options. J. Finance (1998) 1165-1190.

[7] M.H. Davis, V.G. Panas and T. Zariphopoulou, European Option Pricing with Transaction Costs. SIAM J. Control Optim. 3 (1993) 470-493.

[8] E. Derman and I. Kani, Riding on a Smile. Risk Mag. (1994) 32-39.

[9] E. Derman and I. Kani, Stochastic Implied Trees: Arbitrage Pricing with Stochastic Term and Strike Structure of Volatility. Int. J. Theor. Appl. Finance 1 (1998) 61-110.

[10] R. Douady, Closed Form Formulas for Exotic Options and their Lifetime Distribution. Int. J. Theor. Appl. Finance 2 (1998) $17-42$.

[11] N. Dubourg, Couverture dynamique en présence d'imperfections, Ph.D. Thesis. Univ. Paris I (1997).

[12] B. Dupire, Pricing and Hedging with Smiles in Mathematics of Derivative Securities, edited by M.A.H. Dempster and S.R. Pliska. Cambridge Univ. Press, Cambridge (1997) 103-111.

[13] B. Dupire, A Unified Theory of Volatility, Preprint. Paribas Capital Markets (1995).

[14] A. Friedman, Partial differential equations of parabolic type. Prentice-hall, Inc. Englewood Cliffs, N.Y. (1964).

[15] A.V. Fursikov, Lagrange principle for problems of optimal control of ill-posed or singular distributed systems. J. Math. Pures Appl. 71 (1992) 139-194.

[16] A.V. Fursikov and O.Yu. Imanuvilov, On approximate controllability of the Stokes system. Ann. Fac. Sci. Toulouse 11 (1993) 205-232.

[17] A.V. Fursikov and O.Yu. Imanuvilov, Local exact controllability of two dimensional Navier-Stokes system with control on the part of the boundary. Math. Sbornik. 187 (1996).

[18] L. Hörmander, Linear partial differential operators. Springer-Verlag, Berlin (1963).

[19] N. El Karoui, Évaluation et couverture des options exotiques, Working paper. Univ. Paris VI (1997). 
[20] R. Lattès and J.-L. Lions, Méthode de quasi-réversibilité et applications. Dunod, Paris (1967).

[21] R.C. Merton, Theory of Rational Option Pricing. Bell J. Econ. Manag. Sci. 4 (1973) 141-183.

[22] J.-L. Lions, Contrôle optimal de systèmes gouvernés par des équations aux dérivées partielles. Dunod Gauthier-Villars, Paris (1968).

[23] M. Rubinstein, Exotic Options, Finance Working Paper No. 220. U.C. Berkeley (1991).

[24] P.O. Shorygin, On the Controllability Problem Arising in Financial Mathematics. J. Dynam. Control. Syst. 6 (2000) 353-363.

[25] N. Taleb, Dynamic Hedging: Managing Vanilla and Exotic Options. J. Wiley \& Sons, New York (1997).

[26] D. Tataru, Carleman estimates and unique continuation for solutions to boundary value problems. J. Math. Pures Appl. 75 (1996) 367-408.

[27] M.E. Taylor, Partial Differential Equations II. Springer-Verlag, Berlin (1991).

\section{Related papers not cited in the article}

[28] P. Acworth, Pricing and Hedging Barrier and Forward Start Options Using Static Replication, Working paper. ING Barings (1997).

29] S. Allen and O. Padovani, Risk Management Using Static Hedging, Working paper. Courant Institute, N.Y.U. (2001).

[30] L. Andersen and J. Andreasen, Static Barriers. RISK (2000) 120-122.

[31] S. Aparicio and L. Clewlow, A Comparison of Alternative Methods for Hedging Exotic Options, Working paper. FORC (1997).

[32] A. Bhandari, Static Hedging: A Genetic Algorithms Approach. Working paper (1999).

[33] J. Bowie and P. Carr, Static Simplicity. RISK (1994) 44-50.

[34] H. Brown, D. Hobson and C. Rogers, Robust Hedging of Barrier Options. Math. Finance 11 (2000) 285-314.

[35] P. Carr and J. Picron, Static Hedging of Timing Risk. J. Derivatives (1999) 57-66.

[36] P. Carr and A. Chou, Static Hedging of Complex Barrier Options, Working paper. Courant Institute, N.Y.U. (1998).

[37] A. Chou and G. Grigoriev, A Uniform Approach to Static Replication. J. Risk Fall (1998) 73-86.

[38] M. Davis, W. Schachermayer and R. Tompkins, Pricing, No-arbitrage Bounds and Robust Hedging of Installment Options, Working paper. Tech. Univ. Vienna, Austria (2000).

[39] E. Derman, D. Ergener and I. Kani, Forever Hedged RISK (1995) 139-145.

[40] E. Derman, D. Ergener and I. Kani, Static Option Replication. J. Derivatives 2 (1995) 78-85.

[41] N. El Karoui and M. Jeanblanc-Piqué Exotic Options Without Mathematics, Working paper. Univ. Paris VII (1997).

[42] E. Haug, First...Then...Knock-out Options. Wilmott Mag. (2001).

[43] E. Haug, Barrier Put-Call Transformations, Working paper. Paloma Partners (1999).

[44] E. Herzfeld and H. Konishi, Static Replication of Interest Rate Contingent Claims, Master Thesis. M.I.T. (1997).

[45] D. Hobson, Robust Hedging of the Lookback Option. Finance and Stochastics 2 (1998) 329-347.

[46] P. Jaeckel and R. Rebonato, An Efficien and General Method to Value American-style Equity and FX Options in the Presence of User-defined Smiles and Time-dependent Volatility, Working paper. NatWest (1999).

[47] G. Koutmos, Financial Risk Management: Dynamic vs. Static Hedging. Global Bus. Econ. Rev. I (1999) 60-75.

[48] G. Peccati, A Time-space Hedging Theory, Working paper. Univ. Paris VI (2001).

[49] A. Sbuelz, A General Treatment of Barrier Options and Semi-static Hedges of Double Barrier Options, Working paper. Tilburg Univ. (2000).

[50] A. Sbuelz, Semi-static Hedging of Double Barrier Options, Working paper. Tilburg Univ. (2000).

[51] B. Thomas, Exotic Options II in Handbbok of Risk Management, Chap. 4, edited by C. Alexander (1998).

[52] H. Thomsen, Barrier Options: Evaluation and Hedging, Dissertation. Aarhus Univ. (1998).

[53] K. Toft and C. Xuan, How Well Can Barrier Options be Hedged by a Static Portfolio of Standard Options? J. Fin. Engrg. 7 (1998) $147-175$.

[54] R. Tompkins, Static vs. Dynamic Hedging of Exotic Options: An Evaluation of Hedge Performance via Simulation. Net Exposure 2 (1997) 1-36. 